Artigo Original

Original Article

Silmara de Abreu Melgaço ${ }^{1}$ (C) Laelia Cristina Caseiro Vicente ${ }^{2}$ (C) Ana Cristina Côrtes Gama²

Descritores

Neoplasias Bucais

Neoplasias da Língua

Traqueostomia

Glossectomia

Deglutição

Transtornos de Deglutição

Fonoaudiologia

Keywords

Oral Neoplasms

Neoplasms of Tongue

Tracheostomy

Glossectomy

Swallowing

Deglutition Disorders

Speech Therapy

\section{Análise do tempo de decanulação e liberação de via oral em pacientes com câncer de boca}

\author{
Analysis of decannulation time and oral intake \\ recovery in oral cancer patients
}

\author{
Endereço para correspondência: \\ Silmara de Abreu Melgaço \\ Faculdade de Medicina, Universidade \\ Federal de Minas Gerais - UFMG \\ Avenida Alfredo Balena, $\mathrm{n}^{0}$ 190, sala \\ 251, Santa Efigênia, Belo Horizonte \\ (MG), Brasil, CEP: 30.130-100. \\ E-mail: silmelgaco@hotmail.com
}

\begin{abstract}
Purpose: to analyze the time of decannulation and oral diet release of patients undergoing oral cancer surgery at the Hospital Alberto Cavalcante and to verify which factors are associated with the time of decannulation and oral diet release. Methods: an observational study of the database of 33 adult patients surgically treated with oral cancer and served between 2012 and 2017. The socio-demographic variables (age and sex) and clinical variables (type of surgery, surgical extension, type of reconstruction, clinical conditions and times of decannulation and reintroduction of the oral route) were collected through electronic medical records analysis. Descriptive statistical analysis was performed with measures of central tendency, dispersion and proportions. For the association analysis, the non-parametric Mann-Whitney test was used for independent samples. Results: of the 33 participants, male and elderly predominated, $69.8 \%$ underwent resection of more than one structure. The median time of decannulation among patients with oral cancer was 8 days, and oral clearance of 9.5 days. Resections with more than one structure, the presence of fistula and dehiscence interfered in the oral release time. Conclusion: the median time of decannulation was eight days and oral release of 9.5 days. Resections with more than one structure, the presence of fistula, and suture dehiscence are associated with increased oral delivery time.
\end{abstract}

\footnotetext{
Trabalho realizado no Programa de Pós-graduação em Ciências Fonoaudiológicas da Universidade Federal de Minas Gerais - UFMG - Belo Horizonte (MG), Brasil.

${ }^{1}$ Faculdade de Medicina, Universidade Federal de Minas Gerais - UFMG - Belo Horizonte (MG), Brasil.

${ }^{2}$ Departamento de Fonoaudiologia, Faculdade de Medicina, Universidade Federal de Minas Gerais - UFMG Belo Horizonte (MG), Brasil.

Fontes de financiamento: Fundação de Amparo à Pesquisa do Estado de Minas Gerais - Fapemig (APQ-02594-15) Conflito de interesses: nada a declarar.
} 


\section{INTRODUÇÃO}

O câncer de boca, apesar de fácil diagnóstico, vem crescendo nos últimos anos entre os homens brasileiros, com tumores que afetam lábios, língua, assoalho da boca, mandíbula e palato duro, segundo o Instituto Nacional do Câncer (INCA) ${ }^{(1)}$. Estima-se 11.200 novos casos de câncer de boca no Brasil em homens e 4.010 novos casos em mulheres para o ano de $2020^{(1)}$.

O tratamento cirúrgico do câncer de boca pode deixar sequelas como deformidades orofaciais e disfagia orofaríngea, por comprometer uma ou mais estruturas, parcial ou totalmente, acarretando distúrbios nutricionais que levam à piora no estado geral de saúde do paciente ${ }^{(2)}$.

A disfagia pode causar a redução da ingestão de alimentos e mudanças desfavoráveis na dieta, que podem levar à desnutrição, desidratação e diminuição da resistência à infecção $0^{(3)}$. Nestes casos, a utilização de via alternativa de alimentação e de hidratação, como o cateter nasoentérico (CNE), pode ser necessária ${ }^{(3)}$.

Geralmente os pacientes com câncer de boca no pós-operatório permanecem com via alternativa de alimentação por alguns dias, para que a cicatrização da área remanescente ocorra, contudo, a capacidade de deglutição é considerada um aspecto importante na evolução do processo de retirada do $\mathrm{CNE}^{(4)}$.

A traqueostomia também pode estar presente no pós-operatório em alguns casos, por ser uma alternativa temporária para respiração enquanto a área operada estiver edemaciada, obstruindo as vias aéreas superiores ${ }^{(5)}$.

A traqueostomia está associada ao aumento do risco da aspiração e pode gerar impacto mecânico e funcional na fisiologia da deglutição ${ }^{(5)}$. A restrição da excursão hiolaríngea e o desvio do fluxo aéreo para o estoma no pescoço reduz a pressão e a quantidade do fluxo de ar, consequentemente as pregas vocais fecham com menor força, o que pode facilitar a aspiração do bolo alimentar, além do risco de ocorrência de estase, que piora a função de deglutição do indivíduo ${ }^{(5,6)}$. Outro impacto fisiológico decorrente da traqueostomia é a alteração da temperatura do fluxo respiratório nas vias aéreas inferiores, que proporciona a dessensibilização da mucosa e consecutiva aspiração silenciosa ${ }^{(7,8)}$.

A coordenação entre a respiração e a deglutição é importante para que o processo de alimentação ocorra de forma segura. Neste processo, a apneia de deglutição acontece para que ocorra a passagem do bolo alimentar pela faringe até o esôfago, e o fluxo expiratório, ao final da fase faríngea da deglutição, tem a função de limpeza de possíveis resíduos pós-deglutição ${ }^{(9,10)}$. Dessa forma, a presença da traqueostomia pode desencadear modificações na integração das funções respiratórias e de deglutição e, assim, desencadear a disfagia ${ }^{(6)}$, o que justifica a necessidade de decanulação, assim que possível, nos pacientes em que a dinâmica da deglutição está prejudicada.

Portanto, é de suma importância analisar os tempos de decanulação e de liberação de via oral e identificar os fatores que podem influenciar nesses processos em pacientes tratados cirurgicamente do câncer de boca, a fim de se estabelecer balizadores para a reabilitação fonoaudiológica.

O presente estudo tem como objetivo identificar os tempos de decanulação e de liberação da alimentação por meio da via oral em pacientes tratados cirurgicamente do câncer de boca e verificar quais fatores estão associados ao atraso no tempo de decanulação e de liberação de dieta por via oral.

\section{MÉTODO}

Trata-se de um estudo observacional analítico transversal com amostra de conveniência e análise de dados secundários, por meio de prontuário eletrônico de pacientes com diagnóstico de câncer de boca tratados cirurgicamente no Hospital Alberto Cavalcante em Belo Horizonte, MG.

O presente estudo foi aprovado pelos Comitês de Ética em Pesquisa da Fundação Hospitalar do Estado de Minas Gerais e da Universidade Federal de Minas Gerais com os respectivos números: 57666016.9.0000.5119 e 57666016.9.3001.5149, com dispensa do Termo de Consentimento Livre e Esclarecido (TCLE), por ser um estudo observacional com análise de dados secundários.

A amostra foi composta por pacientes com diagnóstico de câncer de boca, tratados cirurgicamente, de ambos os sexos, maiores de 18 anos, atendidos nos setores de Câncer de Cabeça e Pescoço (CCP) e de Fonoaudiologia do Hospital Alberto Cavalcante, referência em Oncologia na Rede Estadual de Saúde. Foram excluídos os indivíduos com ressecções associadas de outras regiões da cabeça e pescoço, doenças neurológicas, distúrbios de comunicação prévios ou alterações cognitivas que impedissem o participante de compreender as instruções fornecidas no processo de avaliação e tratamento fonoaudiológico.

A coleta dos dados da pesquisa ocorreu entre janeiro de 2012 a julho de 2017. Neste período foram atendidos 47 pacientes com diagnóstico de câncer de boca, e deste total, 33 foram inseridos na pesquisa, por se adequarem aos critérios de inclusão e exclusão do estudo.

Durante a internação, a avaliação e a intervenção fonoaudiológica da deglutição e da fala nas glossectomias, pelvectomias, maxilectomias e mandibulectomias inicia-se no segundo dia de pós-operatório, enquanto nas cirurgias combinadas de ressecção de duas ou mais estruturas, no quarto dia de pós-operatório.

A avaliação da fonoarticulação envolve a avaliação perceptivo-auditiva da qualidade da voz (escala GRBASI) (11); da inteligibilidade de fala, tipo articulatório e velocidade de fala (escala de 4 pontos de graus de desvio) ${ }^{(12)}$; medida de tempo máximo de fonação (TMF); e avaliação da ressonância (equilibrada ou nasal) $^{(13)}$.

A avaliação clínica da biomecânica da deglutição é iniciada com saliva e, de acordo com o tipo de abordagem cirúrgica e de reconstrução, é escolhida a consistência mais eficiente e segura para avaliação com alimentos. Para auxiliar no processo de análise da deglutição, são empregados recursos adicionais como a ausculta cervical e monitoramento da saturação de oxigênio ${ }^{(14)}$.

Durante o período de internação, as sessões são realizadas uma vez ao dia, exceto aos finais de semana e feriados. Após a alta hospitalar, o acompanhamento ambulatorial é semanal, com sessões de 40 minutos e a prescrição de programa terapêutico ocorre de acordo com o comprometimento funcional do paciente, a ser seguido diariamente em casa.

Para os casos de câncer de boca geralmente nos programas terapêuticos são realizados exercícios de motricidade orofacial para favorecer a coordenação e a mobilidade das estruturas remanescentes do sistema estomatognático ${ }^{(15)}$, e quando 
necessário, sensibilização laríngea por meio de exercícios vocais e respiratórios. O treino funcional da deglutição é realizado por meio de oferta de alimentos em consistências e volumes específicos, de acordo com a tolerância do paciente, e aumento progressivo, até liberação da via oral segura e eficiente. Quando necessário, são utilizadas as manobras de controle motor oral, de proteção de via aérea e de limpeza de resíduos para facilitar a biomecânica da deglutição ${ }^{(15)}$.

Para caracterização da amostra e definição dos tempos de decanulação e de liberação da alimentação por meio da via oral foram coletados dados nos prontuários dos participantes, obtidos na descrição da cirurgia, e nas evoluções médicas e fonoaudiológicas.

As variáveis sociodemográficas consideradas foram sexo e idade. As variáveis clínicas estudadas compreenderam os tempos de decanulação e de liberação de via oral (VO), ambos em dias; a presença de radioterapia prévia (sim, não); a manutenção de estado de alerta (sim ou não); a presença de doenças respiratórias ( $\operatorname{sim}$ ou não); presença de tosse eficaz ( $\operatorname{sim}$ ou não); e as consistências liberadas, classificadas por meio da escala International Dysphagia Diet Standardisation Initiative (IDSSI): 0 (líquido ralo), 2 (líquido levemente espessado), 3 (líquido moderamente espessado), 4 (pastoso), 6 (sólido macio e picado) e 7 (normal) ${ }^{(16)}$. As variáveis clínicas referentes ao procedimento cirúrgico foram: tipo de cirurgia (glossectomia, pelvectomia, mandibulectomia, palatectomia ou ressecções associadas entre estas estruturas); extensão operatória da língua (parcial ou total); extensão operatória do assoalho de boca (parcial ou total); extensão operatória da mandíbula (marginal, segmentar podendo ser a ressecção anterior, lateral ou lateral e anterior, parcial, quase total ou total); extensão operatória do palato (parcial ou total); tipo de reconstrução da língua e do assoalho de boca (sutura primária com a própria estrutura, retalho regional, retalho livre, não realizado) ${ }^{(17)}$; tipo de reconstrução da mandíbula (retalhos livres ósseos ou placa de titânio) ${ }^{(17)}$; tipo de reconstrução do palato (placa obturadora ou não realizada); presença de esvaziamento cervical (sim ou não); presença de fístula pós operatória ( deiscência de sutura ( $\operatorname{sim}$ ou não); e realização de traqueostomia (TQT) (sim ou não). O estadiamento clínico não foi incluído devido à ausência dessa informação na maioria dos prontuários.

Para análise da associação foram consideradas variáveis de resposta o tempo de decanulação e de liberação de dieta por via oral, ambas em dias. As variáveis explicativas consideradas foram faixa etária, extensão operatória, presença de fístula e presença de deiscência. A variável faixa etária foi categorizada em dois grupos: até 59 anos $(\mathrm{N}=14)$ e acima de 60 anos $(\mathrm{N}=19)$. Para categorização da variável extensão cirúrgica foram formados dois grupos: para o Grupo 1 foram considerados os pacientes submetidos à ressecção de uma única estrutura $(\mathrm{N}=10)$, e para o Grupo 2 os indivíduos submetidos à ressecção de duas ou mais estruturas da cavidade oral $(\mathrm{N}=23)$.

A análise estatística dos dados foi realizada por meio do programa estatístico SPSS (Statistical Package for the Social Sciences) versão 17.0. Primeiramente foi realizada análise descritiva dos dados com medidas de tendência central, dispersão e proporções. Posteriormente, para a análise de associação, foi utilizado o teste não paramétrico Mann-Whitney para amostras independentes. Foi considerado o nível de significância de 5\%.

\section{RESULTADOS}

A amostra foi composta por 33 pacientes que trataram cirurgicamente do câncer de boca, sendo que as características demográficas e clínicas, referentes aos indivíduos são apresentadas na Tabela 1. Observa-se predomínio do sexo masculino e de

Tabela 1. Caracterização demográfica e clínica dos pacientes com câncer de boca

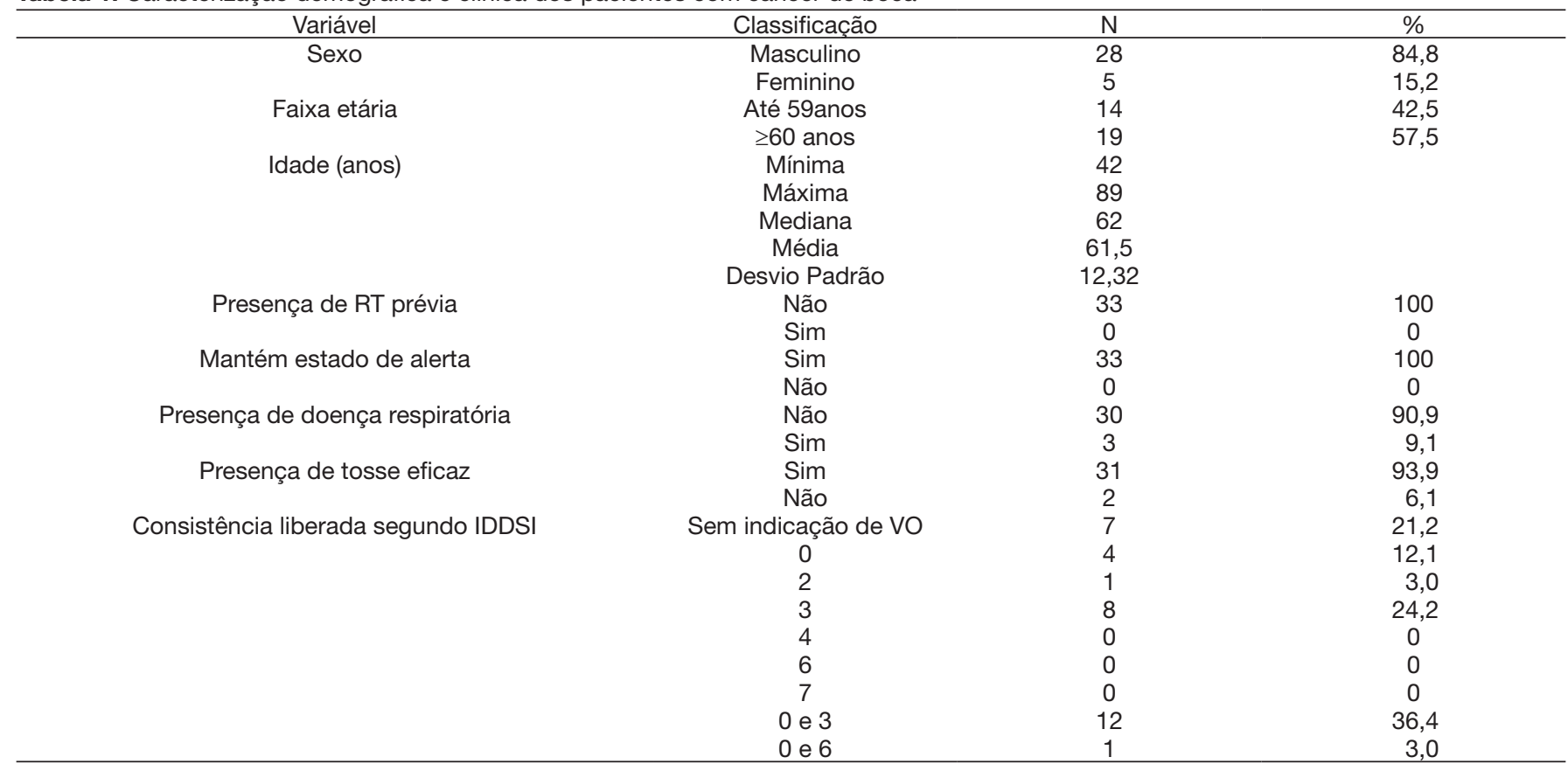

Legenda: $\mathrm{N}$ = número de participantes; RT = radioterapia; IDDSI = International Dysphagia Diet Standardisation Initiative (Iniciativa Internacional de Padronização de Dietas para Disfagia); IDDSI 0 = líquido ralo; IDDSI 2 = líquido levemente espessado; IDDSI 3 = líquido moderamente espessado; IDDSI 4 = pastoso; IDDSI 6 = sólido macio e picado; IDDSI 7 = dieta normal. 
idosos. Nenhum indivíduo realizou radioterapia previamente à cirurgia. Em relação às variáveis clínicas, todos os pacientes estavam alerta, respondendo à comandos e apresentaram tosse eficaz, capaz de mobilizar e expectorar secreção pulmonar. A maioria não possuía diagnóstico de doenças pulmonares e conseguiu a liberação da $\mathrm{VO}$ de alguma consistência $(78,8 \%)$ durante o período de intervenção fonoaudiológica.

As variáveis referentes à cirurgia são apresentadas na Tabela 2. Em relação ao tipo de cirurgia, $69,8 \%$ dos indivíduos realizaram operação combinada com ressecção de mais de uma estrutura. Nos casos de glossectomia e de pelvectomia, a ressecção parcial aconteceu em todos os pacientes. Em relação à extensão da mandibulectomia, a exérese do segmento lateral da mandíbula foi a mais frequente. Houve apenas um caso de ressecção parcial de palato, sem reconstrução. A língua foi a estrutura mais utilizada para a reconstrução do assoalho da boca. Nas cirurgias em que a mandíbula ou o palato foram ressecados, não houve reconstrução. Não ocorreu presença de fístula ou deiscência de sutura no pós-operatório da maioria dos pacientes.

Em relação ao processo de decanulação e liberação de VO, dos 24 pacientes que foram traqueostomizados, 18 indivíduos (75\%) foram decanulados. Quanto à reintrodução da via oral, dos 33 pacientes, 26 indivíduos (78,8\%) tiveram a VO liberada. A mediana do tempo de liberação de $\mathrm{VO}$ foi de 9,5 dias e do tempo de decanulação foi de oito dias (Tabela 3 ).

Não houve associação das variáveis estudadas com o tempo de decanulação (Tabela 4).

Observa-se associação entre o tempo de liberação de via oral e maior extensão operatória, presença de fístula e presença de deiscência (Tabela 5).

Tabela 2. Características do tratamento operatório dos participantes com câncer de boca*

\begin{tabular}{|c|c|c|c|}
\hline Variáveis & Classificação & $\mathrm{N}$ & $\%$ \\
\hline \multirow[t]{6}{*}{ Tipo de cirurgia } & Pelveglossomandibulectomia & 16 & 48,5 \\
\hline & Glossectomia & 7 & 21,2 \\
\hline & Pelveglossectomia & 5 & 15,2 \\
\hline & Mandibulectomia & 2 & 6,1 \\
\hline & Pelvemandibulectomia & 2 & 6,1 \\
\hline & Ressecção de Palato & 1 & 3 \\
\hline \multirow[t]{2}{*}{ Extensão operatória da língua } & Parcial & 28 & 100 \\
\hline & Total & 0 & 0 \\
\hline \multirow[t]{2}{*}{ Extensão operatória do assoalho de boca } & Parcial & 23 & 100 \\
\hline & Total & 0 & 0 \\
\hline \multirow[t]{6}{*}{ Extensão operatória da mandíbula } & Segmentar lateral & 12 & 60 \\
\hline & Segmentar anterior & 2 & 10 \\
\hline & Segmentar anterior e lateral & 2 & 10 \\
\hline & Marginal & 2 & 10 \\
\hline & Quase total & 1 & 3 \\
\hline & Total & 1 & 3 \\
\hline \multirow[t]{2}{*}{ Extensão operatória do palato } & Parcial & 1 & 100 \\
\hline & Total & 0 & 0 \\
\hline \multirow[t]{4}{*}{ Tipo de reconstrução/sutura de língua } & Primário & 27 & 96,4 \\
\hline & Retalho regional & 1 & 3,6 \\
\hline & Retalho livre & 0 & 0 \\
\hline & Não realizada & 0 & 0 \\
\hline \multirow[t]{4}{*}{ Tipo de reconstrução de assoalho de boca } & Retalho regional & 21 & 100 \\
\hline & Primário & 0 & 0 \\
\hline & Retalho livre & 0 & 0 \\
\hline & Não realizada & 0 & 0 \\
\hline \multirow[t]{3}{*}{ Tipo de reconstrução de mandíbula } & Não realizada & 20 & 100 \\
\hline & Retalho livre ósseo & 0 & 0 \\
\hline & Placa de titânio & 0 & 0 \\
\hline \multirow[t]{3}{*}{ Tipo de reconstrução de palato } & Não realizada & 1 & 100 \\
\hline & Retalho livre & 0 & 0 \\
\hline & Placas Obturadoras & 0 & 0 \\
\hline \multirow[t]{2}{*}{ Presença de esvaziamento cervical } & Sim & 28 & 84,8 \\
\hline & Não & 15 & 15,2 \\
\hline \multirow[t]{2}{*}{ Presença de fístula pós-operatória } & Não & 27 & 81,8 \\
\hline & Sim & 6 & 18,2 \\
\hline \multirow[t]{2}{*}{ Presença de deiscência de sutura } & Não & 30 & 90,9 \\
\hline & Sim & 3 & 9,1 \\
\hline \multirow[t]{2}{*}{ Realização de Traqueostomia } & Sim & 24 & 72,7 \\
\hline & Não & 9 & 27,3 \\
\hline
\end{tabular}


Tabela 3. Medidas de tendência central e dispersão para tempo para decanulação e liberação de dieta via oral

\begin{tabular}{|c|c|c|c|c|c|c|c|c|c|}
\hline Variável & $\mathrm{N}$ & Mín & Máx & Mediana & Média & $\mathrm{DP}$ & P25 & P50 & P75 \\
\hline Tempo de decanulação (dias) & 18 & 4 & 140 & 8,0 & 24,50 & 38,80 & 6,75 & 8,0 & 20,3 \\
\hline Tempo de liberação de dieta VO (dias) & 26 & 1 & 347 & 9,5 & 37,00 & 73,10 & 3,75 & 9,5 & 37,3 \\
\hline
\end{tabular}

Legenda: N= número de participantes; Min = Mínima; Máx = Máxima; DP = Desvio Padrão; P = Percentil; VO = Via Oral.

Tabela 4. Associação entre tempo para decanular e faixa etária, extensão operatória, presença de fístula e deiscência

\begin{tabular}{|c|c|c|c|c|c|}
\hline & & $\mathrm{N}$ & Média & $\mathrm{DP}$ & $p$ \\
\hline \multirow[t]{2}{*}{ Faixa etária } & Até 59 anos & 10 & 24,60 & 42,03 & 0,788 \\
\hline & $\geq 60$ anos & 8 & 24,37 & 37,20 & \\
\hline \multirow[t]{2}{*}{ Extensão operatória } & Grupo 1 & 5 & 33,00 & 59,83 & 0,427 \\
\hline & Grupo 2 & 13 & 21,23 & 29,96 & \\
\hline \multirow[t]{2}{*}{ Presença de fístula } & Não & 14 & 19,64 & 29,23 & 0,957 \\
\hline & Sim & 4 & 41,50 & 65,83 & \\
\hline \multirow{2}{*}{$\begin{array}{c}\text { Presença de } \\
\text { deiscência }\end{array}$} & Não & 16 & 24,00 & 4,098 & 0,179 \\
\hline & Sim & 2 & 28,50 & 19,09 & \\
\hline
\end{tabular}

Teste Mann-Whitney

Legenda: DP = Desvio Padrão

Tabela 5. Associação entre tempo para liberação de dieta por via oral e faixa etária, extensão operatória, presença de fístula e deiscência

\begin{tabular}{|c|c|c|c|c|c|}
\hline & & $\mathrm{N}$ & Média & DP & $p$ \\
\hline \multirow[t]{2}{*}{ Faixa etária } & Até 59anos & 11 & 58,55 & 105,98 & 0,466 \\
\hline & $\geq 60$ anos & 15 & 21,20 & 29,78 & \\
\hline \multirow[t]{2}{*}{ Extensão operatória } & Grupo 1 & 9 & 20,44 & 50,9 & 0,013 \\
\hline & Grupo 2 & 17 & 45,76 & 82,57 & \\
\hline \multirow[t]{2}{*}{ Presença de fístula } & Não & 21 & 17,05 & 26,4 & 0,011 \\
\hline & Sim & 5 & 120,80 & 138,01 & \\
\hline \multirow[t]{2}{*}{ Presença de deiscência } & Não & 23 & 35,39 & 77,75 & 0,040 \\
\hline & Sim & 3 & 49,33 & 5,51 & \\
\hline
\end{tabular}

Teste Mann-Whitney

Legenda: DP = Desvio Padrão.

\section{DISCUSSÃO}

Neste estudo a maioria da população com câncer de boca foi do sexo masculino. Segundo estimativas do INCA para o ano de 2020, o câncer de cavidade oral é um dos mais frequentes entre os homens e, nos últimos anos, tem-se observado um aumento na ocorrência entre as mulheres, o que pode ser resultante do aumento dos hábitos de fumar e/ou consumir bebidas alcoólicas ${ }^{(1)}$.

A ressecção cirúrgica continua sendo o principal tratamento para muitos tipos de câncer de boca, porém, a cirurgia compromete a anatomia e as funções de deglutição e de fala, e pode causar comprometimentos funcionais definitivos, apesar dos avanços em abordagens cirúrgicas minimamente invasivas, e das reconstruções microcirúrgicas ${ }^{(18)}$.

A frequência de decanulação entre os pacientes com câncer de boca foi de $75 \%$ e a mediana de tempo de oito dias. Em estudos com casos de glossectomias a frequência foi maior, variou entre $84 \%$ e $90 \%{ }^{(19,20)}$. Já a mediana de tempo de decanulação é inferior a 13,7 dias para pacientes que realizaram hemiglossectomia ${ }^{(21)}$ e superior a 3,5 meses para pacientes que realizaram glossectomia com ressecção de pelo menos $75 \%$ da língua ${ }^{(22)}$. Os resultados encontrados neste estudo podem ser decorrentes da extensão operatória. Embora sem relevância estatística, os casos com ressecções de mais de uma estrutura da cavidade oral são, geralmente, tumores maiores e com pior estadiamento. Estes quadros clínicos tornam o tratamento cirúrgico mais extenso, o que pode causar edema, fistulas e deiscência, e retardar a retirada da traqueostomia.

A frequência da liberação da VO foi de $79 \%$ e a mediana do tempo de liberação de via oral no grupo de pacientes estudados foi de 9,5 dias. Em estudo com pacientes submetidos à glossectomia parcial, subtotal ou total, verificou que a $\mathrm{VO}$ foi conseguida por $49 \%$ dos participantes, enquanto $16 \%$ eram parcialmente dependentes da via alternativa de alimentação e $36 \%$ eram totalmente dependentes da via enteral para nutrição. Nos pacientes que conseguiram VO, a média do tempo foi de 31 dias (variando de 9 - 209 dias) ${ }^{(23)}$. No presente estudo, a frequência da liberação da $\mathrm{VO}$ maior e em menor tempo, provavelmente deve-se a ausência de casos de glossectomia total ou subtotal. O início da reintrodução da via oral pode variar entre os serviços de câncer de cabeça e pescoço, todavia o tempo encontrado neste estudo indica que após a confirmação de ausência de intercorrências pós-operatórias, o paciente está apto para intervenção referente à deglutição, sendo possível liberar VO nas primeiras semanas após o tratamento cirúrgico.

As ressecções de cavidade oral podem prejudicar a fase oral da deglutição e provocar escape posterior de alimento devido à dificuldade do controle motor oral ${ }^{(24)} \mathrm{e}$, consequentemente, risco de aspiração do bolo alimentar. A literatura aponta uma prevalência de 12 a $25 \%$ de aspiração crônica nos casos de 
cirurgia de câncer de boca ${ }^{(18)}$. A gravidade da disfagia pode ser influenciada por uma série de fatores clínicos e a literatura indica que o tamanho da ressecção da língua, o tipo de reconstrução e o estádio do tumor primário afetam a deglutição ${ }^{(10,23,25)}$.

Neste estudo, a maioria dos pacientes teve as consistências líquida rala (IDSSI 0) e líquida moderamente espessada (IDSSI 3) liberadas. Infere-se que os líquidos ralos e moderadamente espessos são mais fáceis de realizar a ejeção e o controle motor oral respectivamente, principalmente nos casos de limitação na mobilidade das estruturas remanescentes, principalmente nas resseções associadas com mais estruturas. Em um estudo sobre sintomas de disfagia, foi relatado que pacientes tratados cirurgicamente para o câncer de boca, percebem maior grau de dificuldade de deglutição com alimentos sólidos ${ }^{(10)}$. Outro estudo verificou que a deglutição de líquido foi pior no primeiro mês de pós-operatório nos casos com ressecção de 50 a $75 \%$ da língua, devido ao controle motor oral ruim, com aumento de risco de penetrações laríngeas e necessidade de múltiplas deglutições para limpeza de resíduo ${ }^{(24)}$.

Houve associação significativa entre a presença de maior extensão operatória, presença de fístula e de deiscência com o tempo de liberação de via oral. A média de tempo da liberação da dieta por via oral no grupo de pacientes com mais de uma estrutura ressecada (Grupo 2) foi o dobro daqueles com resseções restritas a uma única estrutura (Grupo 1). No que se refere ao tratamento operatório, as alterações de deglutição são multifatorias, variam de acordo com o local e o tamanho do tumor, a extensão da ressecção, o tipo de reconstrução e tratamentos complementares ${ }^{(3,17,23,26)}$. Em geral, quanto maior a ressecção, mais prejudicada será a função da deglutição ${ }^{(17,23)}$.

Vale destacar que a ressecção da língua é uma estrutura importante para a formação e transporte do bolo alimentar e proteção das vias aéreas, desta forma, a ressecção mesmo que parcial pode impactar na biomecânica da deglutição ${ }^{(3)}$. Em estudo de revisão sistemática com glossectomia total e preservação da laringe, identificou a necessidade de via alternativa de alimentação de 0 a $87 \%$ dos casos após seis meses de pós-operatório e de 0 a $75 \%$ após um ano do tratamento cirúrgico ${ }^{(27)}$.

Outras estruturas da cavidade oral ressecadas também são apontadas como causadoras de comprometimentos de mastigação e deglutição. Um exemplo é a ressecção de palato, na qual a cavidade oral mantém comunicação com a cavidade nasal, sendo necessária a reconstrução dessas estruturas para restaurar a função oral ${ }^{(28)}$. Desta forma, quando mais de uma estrutura é ressecada, a reabilitação da deglutição exige mais tempo para promover adaptações e compensações, a fim de se obter função eficiente e segura.

As fístulas e deiscências são complicações pós-operatórias e nesses casos deve-se aguardar reparar o quadro clínico para reintrodução alimentar por $\mathrm{VO}$, desta forma, o uso do $\mathrm{CNE}$ deve ser a única forma de alimentação ${ }^{(18)}$.

Outros fatores são apontados como variáveis que influenciam no sucesso para conseguir a VO nas glossectomias, sendo eles ressecção parcial da língua e ausência de excesso de peso (índice de massa corporal), radioterapia prévia ao tratamento cirúrgico e quimioterapia adjuvante ${ }^{(23)}$.

A reabilitação da deglutição após a cirurgia do câncer é individualizada para atender às necessidades exclusivas de cada paciente. Em geral, pacientes com câncer de boca que realizaram cirurgia (e muitas vezes radioterapia pós-operatória) necessitam reabilitação para fala e deglutição, devendo ser planejada a partir das alterações fisiológicas e funcionais identificadas ${ }^{(18)}$. Para tanto, é importante que o fonoaudiólogo saiba exatamente qual extensão operatória e tipo de reconstrução foi utilizada, a fim de que possa fazer uma avaliação adequada e traçar o programa terapêutico, pois cirurgias classificadas com o mesmo nome podem causar alterações fonoaudiológicas diferentes.

Poucos são os estudos que tratam do tempo de decanulação e liberação de dieta por via oral em pacientes tratados cirurgicamente do câncer de boca, bem como os fatores que podem influenciar esses tempos.

Embora os achados desta pesquisa sejam provenientes de análise secundária em banco de dados, os resultados encontrados apontam para aspectos relevantes que podem subsidiar a atuação fonoaudiológica para a definição de prognóstico e de planejamento terapêutico.

Este estudo apresentou limitações como a ausência do estadiamento clínico do tumor, impossibilitando a análise de associação com as variáveis respostas, além da falta de avaliação instrumental, que poderia contribuir para a análise da biomecânica da deglutição antes e após a intervenção fonoaudiológica nos casos de câncer de boca. O serviço onde foi realizado o estudo não possui os recursos de videofluoroscopia e de videoendoscopia da deglutição.

Estudos futuros com delineamento longitudinal são fundamentais para se estabelecer balizadores do processo terapêutico de pacientes tratados cirurgicamente do câncer de boca.

\section{CONCLUSÃO}

Pacientes submetidos à cirurgia do câncer de boca apresentaram mediana de tempo de decanulação de oito dias e de liberação de via oral de 9,5 dias. As ressecções com mais de uma estrutura e a presença de fístula ou de deiscência de sutura estão associadas ao aumento do tempo de liberação de dieta por via oral.

\section{REFERÊNCIAS}

1. INCA: Instituto Nacional do Câncer [internet]. Brasília: INCA/Ministério da Saúde; 2021. [citado em 2020 Maio 1]. Disponível em: https://www. inca.gov.br/numeros-de-cancer

2. Silva PSL, Leão VML, Scarpel RD. Caracterização da população portadora de câncer de boca e orofaringe atendida no setor de cabeça e pescoço em hospital de referência na cidade de Salvador - BA. Rev CEFAC. 2009;11(3):441-7.

3. Raber-Durlacher JE, Brennan MT, Verdonck-de Leeuw IM, Gibson RJ, Eilers JG, Waltimo T, et al. Swallowing dysfunction in cancer patients. Support Care Cancer. 2012;20(3):433-43. http://dx.doi.org/10.1007/s00520011-1342-2. PMid:22205548.

4. Teixeira, KKR. Abordagem do paciente traqueostomizado no processo de desmame e decanulação [dissertação de mestrado]. Brasília: Sociedade Brasileira de Terapia Intensiva; 2014.

5. Jung SJ, Kim DY, Kim YW, Koh YW, Joo SY, Kim ES. Effect of decannulation on pharyngeal and laryngeal movement in post-stroke tracheostomized patients. Ann Rehabil Med. 2012;36(3):356-64. http:// dx.doi.org/10.5535/arm.2012.36.3.356. PMid:22837971. 
6. Barros, APB; Portas, JG; Queija, DS. Implicações da traqueostomia na comunicação e deglutição. Rev Bras Cir Cabeça Pescoço. 2009;38(3):202-7.

7. Gross RD, Mahlmann J, Grayhack JP. Physiologic effects of open and closed tracheostomy tubes on the pharyngeal swallow. Ann Otol Rhinol Laryngol. 2003;112(2):143-52. http://dx.doi.org/10.1177/000348940311200207. PMid:12597287.

8. O'Connor HH, White AC. Tracheostomy decannulation. Respir Care. 2010;55(8):1076-81. PMid:20667155.

9. Leder SB, Joe JK, Hill SE, Traube M. Effect of tracheotomy tube occlusion on upper esophageal sphincter and pharyngeal pressures in aspirating and nonaspirating patients. Dysphagia. 2001;16(2):79-82. http://dx.doi. org/10.1007/PL00021294. PMid:11305225.

10. Selley WG, Flack FC, Ellis RE, Brooks WA. Respiratory patterns associated with swallowing: part 2. neurologically impaired dysphagic patients. Age Ageing. 1989;18(3):173-6. http://dx.doi.org/10.1093/ageing/18.3.173. PMid:2782214.

11. Hirano M. Clinical examination of voice. New York: Springer Verlag; 1981. p. 81-4.

12. Fouquet ML, Amaral TCRDM, Vicente LCC. Inteligibilidade de fala em pacientes com ressecção de tumor de cavidade de boca e/ou orofaringe. In: Barros, APB. et al., editores. Fonoaudiologia em Cancerologia. São Paulo: Fundação Oncocentro de São Paulo - Comitê de Fonoaudiologia em Cancerologia; 2000. p. 195-204

13. Behlau M. Voz: o livro do especialista. Rio de Janeiro: Revinter; 2001.

14. Padovani AR, Moraes DP, Mangili LD, Andrade CRF. Protocolo fonoaudiológico de avaliação do risco para disfagia (PARD). Rev Soc Bras Fonoaudiol. 2007;12(3):199-205. http://dx.doi.org/10.1590/S151680342007000300007.

15. Hsiang CC, Chen AW, Chen $\mathrm{CH}$, Chen MK. Early postoperative oral exercise improves swallowing function among patients with oral cavity cancer: a randomized controlled trial. Ear Nose Throat J. 2019;98(6):E7380. http://dx.doi.org/10.1177/0145561319839822. PMid:31088304.

16. Cichero JA, Lam P, Steele CM, Hanson B, Chen J, Dantas RO, et al. Development of international terminology and definitions for texturemodified foods and thickened fluids used in dysphagia management: the IDDSI framework. Dysphagia. 2017;32(2):293-314. http://dx.doi. org/10.1007/s00455-016-9758-y. PMid:27913916.

17. Vincent A, Kohlert S, Lee TS, Inman J, Ducic Y. Free-Flap reconstruction of the tongue. Semin Plast Surg. 2019;33(1):38-45. http://dx.doi. org/10.1055/s-0039-1677789. PMid:30863211.

18. Hutcheson KA, Lewin JS. Functional assessment and rehabilitation: how to maximize outcomes. Otolaryngol Clin North Am. 2013;46(4):657-70. http://dx.doi.org/10.1016/j.otc.2013.04.006. PMid:23910476.

19. Rihani J, Lee MR, Lee T, Ducic Y. Flap selection and functional outcomes in total glossectomy with laryngeal preservation. Otolaryngol Head Neck
Surg. 2013;149(4):547-53. http://dx.doi.org/10.1177/0194599813498063. PMid:23884285.

20. Vega C, León X, Cervelli D, Pons G, López S, Fernández M, et al. Total or subtotal glossectomy with microsurgical reconstruction: functional and oncological results. Microsurgery. 2011;31(7):517-23. http://dx.doi. org/10.1002/micr.20922. PMid:21953944.

21. Haughey BH, Taylor SM, Fuller D. Fasciocutaneous flap reconstruction of the tongue and floor of mouth: outcomes and techniques. Arch Otolaryngol Head Neck Surg. 2002;128(12):1388-95. http://dx.doi.org/10.1001/ archotol.128.12.1388. PMid:12479726.

22. Blyth KM, McCabe P, Heard R, Clark J, Madill C, Ballard KJ. Cancer of tongue and floor of mounth: five-year file audit within the acute phase. Am J Speech Lang Pathol. 2014;23(4):668-78. http://dx.doi.org/10.1044/2014_ AJSLP-14-0003. PMid:25089517.

23. Chen DW, Wang T, Shey-Sen Ni J, Sandulache VC, Graboyes EM, Worley $\mathrm{M}$, et al. Prognostic factors associated with achieving total oral diet after glossectomy with microvascular free tissue transfer reconstruction. Oral Oncol. 2019;92:59-66. http://dx.doi.org/10.1016/j.oraloncology.2019.03.005. PMid:31010625.

24. Brown L, Rieger JM, Harris J, Seikaly H. A longitudinal study of functional outcomes after surgical resection and microvascular reconstruction for oral cancer: tongue mobility and swallowing function. J Oral Maxillofac Surg. 2010;68(11):2690-700. http://dx.doi.org/10.1016/j.joms.2010.05.004. PMid:20739112.

25. Oliveira FP, Santos A, Viana MS, Alves JL, Pinho NB, Reis PF. Perfil Nutricional de pacientes com câncer de cavidade oral em pré-tratamento antineoplásico. Rev Bras Cancerol. 2015;61(3):253-9. http://dx.doi. org/10.32635/2176-9745.RBC.2015v61n3.255.

26. Lam L, Samman N. Speech and swallowing following tongue cancer surgery and free flap reconstruction-a systematic review. Oral Oncol. 2013;49(6):507-24. http://dx.doi.org/10.1016/j.oraloncology.2013.03.001. PMid:23566773.

27. Dziegielewski PT, Ho ML, Rieger J, Singh P, Langille M, Harris JR, et al. Total glossectomy with laryngeal preservation and free flap reconstruction: objective functional outcomes and systematic review of the literature. Laryngoscope. 2013;123(1):140-5. http://dx.doi.org/10.1002/lary.23505. PMid:22952109.

28. Murphy BA, Gilbert J. Dysphagia in head and neck cancer patients treated with radiation: assessment, sequelae and rehabilitation. Semin Radiat Oncol. 2009;19(1):35-42. http://dx.doi.org/10.1016/j.semradonc.2008.09.007. PMid:19028344.

\section{Contribuições dos autores}

SAM coletou e analisou os dados e organizou o texto; $L C C V$ e ACCG revisaram criticamente o conteúdo do manuscrito. 